Acta Crystallographica Section E

Structure Reports

Online

ISSN 1600-5368

\section{2-Acetamido- $N$-benzyl-1,4-imino-1,2,4- trideoxy-L-ribitol}

The relative configuration of the stereocentres in a potential hexosaminidase inhibitor, $\mathrm{C}_{14} \mathrm{H}_{20} \mathrm{~N}_{2} \mathrm{O}_{3}$, prepared from D-lyxonolactone, has been established using $\mathrm{X}$-ray crystallographic techniques.

\section{Comment}

Imino sugars, analogues of carbohydrates with the $\mathrm{O}$ atom of the ring replaced by an $\mathrm{N}$ atom, are a family of both natural products and synthetic materials which inhibit glycosidases; several such compounds have considerable therapeutic potential (Watson et al., 2001; Asano et al., 2000; Winchester \& Fleet, 2000). For example, the natural product deoxynojirimycin, (1), is an inhibitor of a range of $\alpha$-glucosidases and its derivatives have been shown to possess antiviral activity (Stütz, 1999); several related pyrrolidines, (2), are also potent inhibitors of $\alpha$-glucosidases, although structure-activity relationships are not easily predictable (Asano et al., 2005; Yu et al., 2004; Scofield et al., 1986). The synthetic $N$-acetylglucosamine analogue, (3), is a powerful hexosaminidase inhibitor (Fleet et al., 1986; Boshagen et al., 1987); such inhibitors have potential as anticancer agents (Woynarowska et al., 1992) and for the treatment of other diseases (Liu et al., 2004). By analogy with the glucosidase inhibitors, (2), a synthetic programme towards a series of diastereomeric pyrrolidines, (4), has led to the preparation of the potential hexosaminidase inhibitor, (5). While the absolute configuration of (5) is established by the use of D-lyxonolactone, (6), as the starting material, ambiguity in the relative configuration of the nitrogen substituent was removed by X-ray crystallographic analysis.
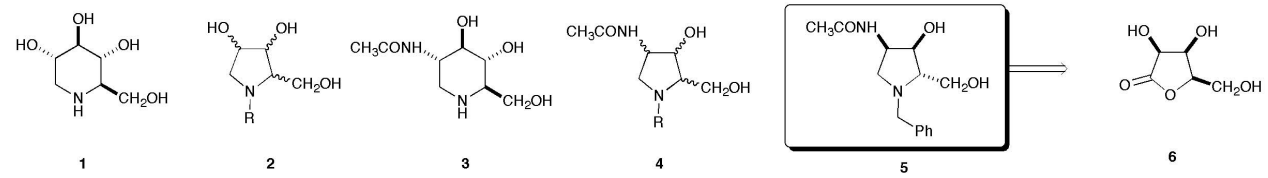

\section{Experimental}

The title compound was crystallized by cooling a warm solution in acetonitrile, forming clear block-like crystals.

Crystal data

$$
\begin{aligned}
& \mathrm{C}_{14} \mathrm{H}_{20} \mathrm{~N}_{2} \mathrm{O}_{3} \\
& M_{r}=264.32 \\
& \text { Monoclinic, } P 2_{1} \\
& a=6.8912(3) \AA \\
& b=7.3504(3) \AA \\
& c=13.6824(6) \AA \\
& \beta=90.822(2)^{\circ} \\
& V=692.98(5) \AA^{3} \\
& Z=2
\end{aligned}
$$

Received 28 February 2005 Accepted 4 March 2005 Online 11 March 2005 


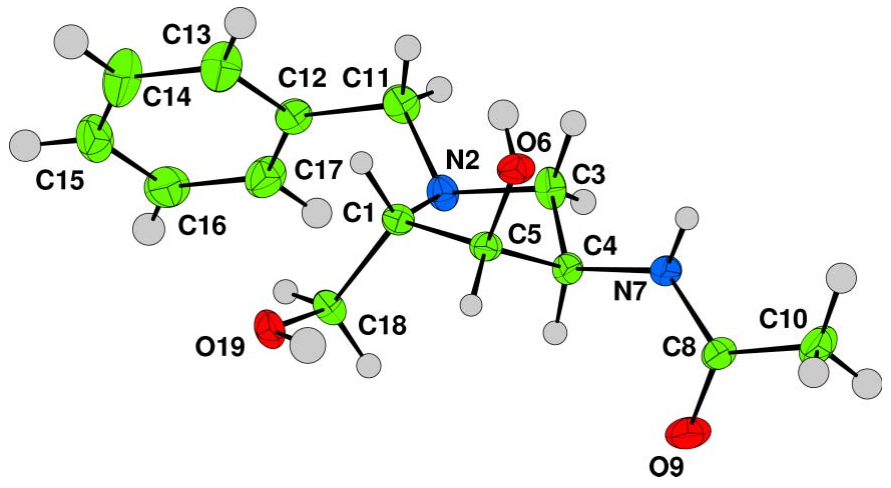

Figure 1

The molecular structure of the title compound, with displacement ellipsoids drawn at the $50 \%$ probability level.

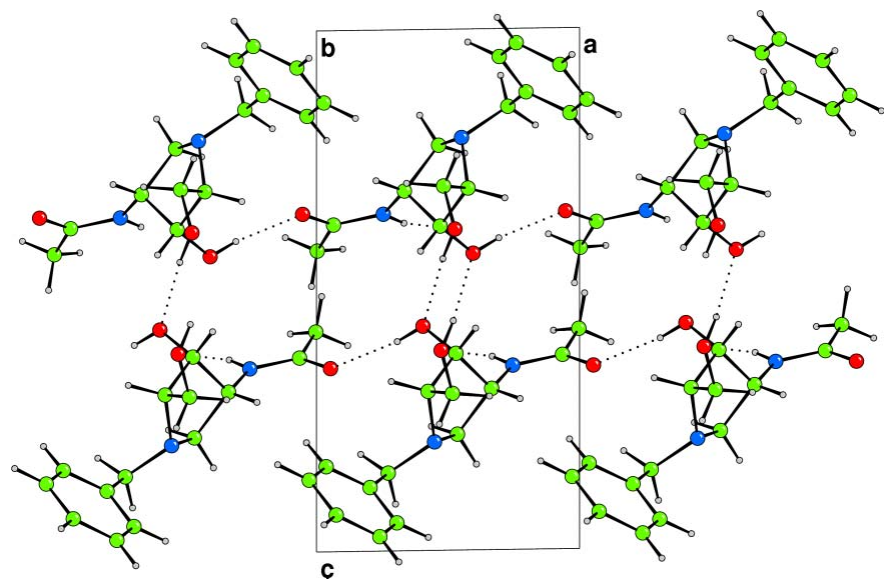

Figure 2

Packing diagram, viewed down the $b$ axis. The crystal structure consists of strongly hydrogen-bonded ribbons of molecules along the $b$ axis, held together by a mixture of hydrogen bonding along the $a$ axis and weaker intermolecular interactions. Hydrogen bonds are represented as dotted lines.

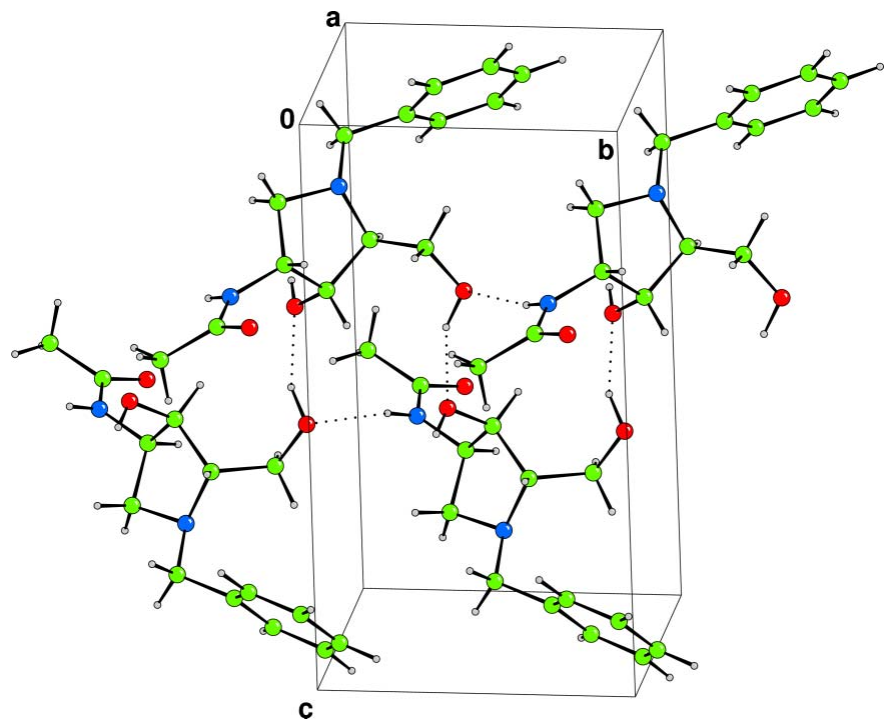

Figure 3

View of the strong hydrogen-bonding network in one of the ribbons running parallel to the $b$ axis. Hydrogen bonds are represented as dotted lines.
Data collection

Nonius KappaCCD diffractometer $\omega$ scans

Absorption correction: multi-scan (DENZO/SCALEPACK

Otwinowski \& Minor, 1997)

$T_{\min }=0.98, T_{\max }=0.99$

2636 measured reflections

Refinement

Refinement on $F^{2}$

$R\left[F^{2}>2 \sigma\left(F^{2}\right)\right]=0.034$

$w R\left(F^{2}\right)=0.086$

$S=0.89$

1673 reflections

172 parameters

$\mathrm{H}$-atom parameters constrained

$w=\left[1-\left(F_{\mathrm{o}}-F_{\mathrm{c}}\right)^{2} / 36 \sigma^{2}\left(F_{\mathrm{o}}\right)\right]^{2 /}$

$\left[33.1 T_{0}(x)+52.7 T_{1}(x)\right.$
1681 independent reflections 1499 reflections with $I>2 \sigma(I)$

$R_{\text {int }}=0.020$

$\theta_{\text {max }}=27.5^{\circ}$

$h=-8 \rightarrow 8$

$l=-17 \rightarrow 17$

$+30.8 T_{2}(x)+12.9 T_{3}(x)$

$\left.+3.03 T_{4}(x)\right]$, where $x=F_{\mathrm{c}} / F_{\max }$ and $T_{i}(x)$ are Chebychev polynomials (Watkin, 1994; Prince, 1982)

$(\Delta / \sigma)_{\max }<0.001$ 。

$\Delta \rho_{\min }=-0.20 \mathrm{e}^{-3}$ $k=-9 \rightarrow 8$

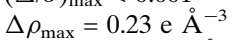

Table 1

Hydrogen-bond geometry $\left(\AA{ }^{\circ}\right)$.

\begin{tabular}{|c|c|c|c|c|}
\hline$D-\mathrm{H} \cdots A$ & $D-\mathrm{H}$ & $\mathrm{H} \cdots A$ & $D \cdots A$ & $D-\mathrm{H} \cdots A$ \\
\hline $\mathrm{N} 7-\mathrm{H} 8 \cdots \mathrm{O} 19^{\mathrm{i}}$ & 0.84 & 2.14 & $2.958(2)$ & 167 \\
\hline $\mathrm{O} 19-\mathrm{H} 15 \cdots \mathrm{O}^{\mathrm{ii}}$ & 0.93 & 1.85 & $2.708(2)$ & 153 \\
\hline $\mathrm{O} 6-\mathrm{H} 17 \cdots \mathrm{O} 9^{\mathrm{iii}}$ & 0.80 & 1.89 & $2.685(2)$ & 168 \\
\hline
\end{tabular}

Symmetry codes: (i) $x, y-1, z$; (ii) $-x+1, y+\frac{1}{2},-z+1$; (iii) $x-1, y, z$.

All $\mathrm{H}$ atoms were observed in a difference electron-density map. The hydroxy and amide $\mathrm{H}$ atoms were refined freely, whilst the others were refined with slack restraints to optimize the geometry. They were all then made to ride on their parent atoms, with $\mathrm{C}-\mathrm{H}$ distances of $0.96-1.00 \AA$ and $U_{\text {iso }}(\mathrm{H})=1.2 U_{\text {eq }}$ (parent). In the absence of significant anomalous scattering effects, Friedel pairs were merged; the absolute configuration is known from the synthesis. Eight lowangle reflections were omitted from the refinement because they appeared to be obscured by the beamstop.

Data collection: COLLECT (Nonius, 1997); cell refinement: DENZO/SCALEPACK (Otwinowski \& Minor, 1997); data reduction: DENZO/SCALEPACK; program(s) used to solve structure: SIR92 (Altomare et al., 1994); program(s) used to refine structure: CRYSTALS (Betteridge et al., 2003); molecular graphics: CAMERON (Watkin et al., 1996); software used to prepare material for publication: CRYSTALS.

\section{References}

Altomare, A., Cascarano, G., Giacovazzo, G., Guagliardi, A., Burla, M. C., Polidori, G. \& Camalli, M. (1994). J. Appl. Cryst. 27, 435.

Asano, N., Ikeda, K., Yu, L., Kato, A., Takebayashi, K., Adachi, I., Kato, I., Ouchi, H., Takahata, H. \& Fleet, G. W. J. (2005). Tetrahedron Asymmetry, 16, 223-229.

Asano, N., Nash, R. J, Molyneux, R. J. \& Fleet, G. W. J. (2000). Tetrahedron Asymmetry, 11, 1645-1680.

Betteridge, P. W., Carruthers, J. R., Cooper, R. I., Prout, K. \& Watkin, D. J. (2003). J. Appl. Cryst. 36, 1487.

Boshagen, H., Heiker, F. \& Schuller, A. (1987). Carbohydr. Res. 164, 141-148.

Fleet, G. W. J., Smith, P. W., Nash, R. J., Fellows, L. E., Parekh, R. B. \& Rademacher, T. W. (1986). Chem. Lett. pp. 1051-1054.

Liu, J. J., Numa, M. M. D., Liu, H. T., Huang, S. J., Sears, P., Shikhman, A. R. \& Wong, C. H. (2004). J. Org. Chem. 69, 6273-6283.

Nonius (1997). COLLECT. Nonius BV, Delft, The Netherlands.

Otwinowski, Z. \& Minor, W. (1997). Methods in Enzymology, Vol. 276, Macromolecular Crystallography, Part A, edited by C. W. Carter Jr \& R. M. Sweet, pp. 307-326. New York: Academic Press. 


\section{organic papers}

Prince, E. (1982). Mathematical Techniques in Crystallography and Materials Science. New York: Springer-Verlag.

Scofield, A. M., Fellows, L. E., Nash, R. J. \& Fleet, G. W. J. (1986). Life Sci. 39, 645-651.

Stütz, A. E. (1999). Iminosugars as Glycosidase Inhibitors: Nojirimycin and Beyond. Weinheim: Wiley-VCH.

Watkin, D. J. (1994). Acta Cryst. A50, 411-437.

Watkin, D. J., Prout, C. K. \& Pearce, L. J. (1996). CAMERON. Chemical Crystallography Laboratory, Oxford, England.
Watson, A. A., Fleet, G. W. J., Asano, N., Molyneux, R. J. \& Nash, R. J. (2001). Phytochemistry, 56, 265-295.

Winchester, B. \& Fleet, G. W. J. (2000). J. Carbohydr. Chem. 19, 471483.

Woynarowska, B., Wilkiel, H., Sharma, M., Carpenter, N., Fleet, G. W. J \& Bernacki, R. J. (1992). Anticancer Res. 12, 161-166.

Yu, C.-Y., Asano, N., Ikeda, K., Wang, M.-X., Butters, T. D., Wormald, M. R., Dwek, R. A., Winters, A. L., Nash, R. J. \& Fleet, G. W. J. (2004). Chem. Commun. pp. 1936-1937. 


\section{supporting information}

Acta Cryst. (2005). E61, o930-o932 [https://doi.org/10.1107/S1600536805006926]

\section{2-Acetamido-N-benzyl-1,4-imino-1,2,4-trideoxy-L-ribitol}

Christopher C. Harding, David J. Watkin, J. S. Shane Rountree, Terry D. Butters, Mark R. Wormald, Raymond A. Dwek and George W. J. Fleet

2-Acetamido-N-benzyl-1,4-imino-1,2,4-trideoxy-L-ribitol

Crystal data

$\mathrm{C}_{14} \mathrm{H}_{20} \mathrm{~N}_{2} \mathrm{O}_{3}$

$M_{r}=264.32$

Monoclinic, $P 2_{1}$

Hall symbol: $\mathrm{P} 2 \mathrm{yb}$

$a=6.8912(3) \AA$

$b=7.3504(3) \AA$

$c=13.6824(6) \AA$

$\beta=90.822(2)^{\circ}$

$V=692.98(5) \AA^{3}$

$Z=2$

\section{Data collection}

Nonius KappaCCD diffractometer

Graphite monochromator

$\omega$ scans

Absorption correction: multi-scan

(DENZO/SCALEPACK; Otwinowski \& Minor, 1997)

$T_{\min }=0.98, T_{\max }=0.99$

\section{Refinement}

Refinement on $F^{2}$

Least-squares matrix: full

$R\left[F^{2}>2 \sigma\left(F^{2}\right)\right]=0.034$

$w R\left(F^{2}\right)=0.086$

$S=0.89$

1673 reflections

172 parameters

1 restraint

Primary atom site location: structure-invariant direct methods
$F(000)=284$

$D_{\mathrm{x}}=1.267 \mathrm{Mg} \mathrm{m}^{-3}$

Mo $K \alpha$ radiation, $\lambda=0.71073 \AA$

Cell parameters from 1415 reflections

$\theta=1-27^{\circ}$

$\mu=0.09 \mathrm{~mm}^{-1}$

$T=190 \mathrm{~K}$

Block, colourless

$0.20 \times 0.20 \times 0.10 \mathrm{~mm}$

2636 measured reflections

1681 independent reflections

1499 reflections with $I>2 \sigma(I)$

$R_{\text {int }}=0.020$

$\theta_{\max }=27.5^{\circ}, \theta_{\min }=1.5^{\circ}$

$h=-8 \rightarrow 8$

$k=-9 \rightarrow 8$

$l=-17 \rightarrow 17$

Hydrogen site location: inferred from neighbouring sites

$\mathrm{H}$-atom parameters constrained $w=\left[1-\left(F_{\mathrm{o}}-F_{\mathrm{c}}\right)^{2} / 36 \sigma^{2}\left(F_{\mathrm{o}}\right)\right]^{2} /\left[33.1 \mathrm{~T}_{0}(\mathrm{x})+\right.$ $\left.52.7 \mathrm{~T}_{1}(\mathrm{x})+30.8 \mathrm{~T}_{2}(\mathrm{x})+12.9 \mathrm{~T}_{3}(\mathrm{x})+3.03 \mathrm{~T}_{4}(\mathrm{x})\right]$, where $\mathrm{x}=F_{\mathrm{c}} / F_{\max }$ and $\mathrm{T}_{\mathrm{i}}(\mathrm{x})$ are Chebychev polynomials (Watkin, 1994; Prince, 1982)

$(\Delta / \sigma)_{\max }=0.000293$

$\Delta \rho_{\max }=0.23$ e $\AA^{-3}$

$\Delta \rho_{\min }=-0.20$ e $\AA^{-3}$

Fractional atomic coordinates and isotropic or equivalent isotropic displacement parameters $\left(\AA^{2}\right)$

\begin{tabular}{lllll}
\hline & $x$ & $y$ & $z$ & $U_{\text {iso }} * / U_{\text {eq }}$ \\
\hline $\mathrm{C} 1$ & $0.4242(3)$ & $0.6215(3)$ & $0.69450(14)$ & 0.0199 \\
$\mathrm{~N} 2$ & $0.4490(3)$ & $0.5333(2)$ & $0.79181(12)$ & 0.0222
\end{tabular}




$\begin{array}{lllll}\text { C3 } & 0.5356(3) & 0.3527(3) & 0.77759(15) & 0.0262 \\ \text { C4 } & 0.6657(3) & 0.3880(3) & 0.69059(14) & 0.0217 \\ \text { C5 } & 0.5299(3) & 0.4963(3) & 0.62235(14) & 0.0195 \\ \text { O6 } & 0.40451(19) & 0.3757(2) & 0.56955(10) & 0.0235 \\ \text { N7 } & 0.7464(2) & 0.2265(3) & 0.64659(13) & 0.0238 \\ \text { C8 } & 0.9353(3) & 0.2105(3) & 0.62727(14) & 0.0231 \\ \text { O9 } & 1.0531(2) & 0.3314(3) & 0.64842(13) & 0.0350 \\ \text { C10 } & 0.9973(3) & 0.0385(4) & 0.57773(18) & 0.0353 \\ \text { C11 } & 0.2725(3) & 0.5279(3) & 0.85049(15) & 0.0271 \\ \text { C12 } & 0.2036(3) & 0.7160(3) & 0.87761(14) & 0.0244 \\ \text { C13 } & 0.0365(4) & 0.7877(4) & 0.83651(18) & 0.0377 \\ \text { C14 } & -0.0277(4) & 0.9605(4) & 0.8619(2) & 0.0460 \\ \text { C15 } & 0.0746(4) & 1.0630(4) & 0.92943(19) & 0.0392 \\ \text { C16 } & 0.2405(4) & 0.9911(4) & 0.9721(2) & 0.0401 \\ \text { C17 } & 0.3054(3) & 0.8199(4) & 0.94605(18) & 0.0341 \\ \text { C18 } & 0.5168(3) & 0.8102(3) & 0.69904(14) & 0.0231 \\ \text { O19 } & 0.4740(2) & 0.9199(2) & 0.61627(11) & 0.0260 \\ \text { H11 } & 0.2843 & 0.6306 & 0.6748 & 0.0234^{*} \\ \text { H31 } & 0.4369 & 0.2583 & 0.7617 & 0.0301^{*} \\ \text { H32 } & 0.6081 & 0.3157 & 0.8355 & 0.0296^{*} \\ \text { H41 } & 0.7736 & 0.4659 & 0.7110 & 0.0263^{*} \\ \text { H51 } & 0.6045 & 0.5678 & 0.5745 & 0.0223^{*} \\ \text { H101 } & 1.1185 & -0.0062 & 0.6045 & 0.0424^{*} \\ \text { H102 } & 1.0191 & 0.0636 & 0.5097 & 0.0433^{*} \\ \text { H103 } & 0.9008 & -0.0577 & 0.5807 & 0.0425^{*} \\ \text { H111 } & 0.1680 & 0.4661 & 0.8139 & 0.0314^{*} \\ \text { H112 } & 0.3003 & 0.4585 & 0.9100 & 0.0306^{*} \\ \text { H131 } & -0.0382 & 0.7154 & 0.7899 & 0.0452^{*} \\ \text { H141 } & -0.1480 & 1.0100 & 0.8313 & 0.0545^{*} \\ \text { H151 } & 0.0289 & 1.1840 & 0.9480 & 0.0462^{*} \\ \text { H161 } & 0.3130 & 1.0614 & 1.0212 & 0.0478^{*} \\ \text { H171 } & 0.4252 & 0.7699 & 0.9749 & 0.0416^{*} \\ \text { H181 } & 0.4672 & 0.8727 & 0.7581 & 0.0277^{*} \\ \text { H182 } & 0.6578 & 0.7973 & 0.7054 & 0.0276^{*} \\ \text { H8 } & 0.6680 & 0.1454 & 0.6289 & 0.0246^{*} \\ \text { H15 } & 0.5200 & 0.8675 & 0.5983 & 0.0425^{*} \\ \text { H17 } & 0.3069 & & \end{array}$

Atomic displacement parameters $\left(\AA^{2}\right)$

\begin{tabular}{lllllll}
\hline & $U^{11}$ & $U^{22}$ & $U^{33}$ & $U^{12}$ & $U^{13}$ & $U^{23}$ \\
\hline C1 & $0.0200(9)$ & $0.0199(10)$ & $0.0198(9)$ & $-0.0007(8)$ & $0.0015(7)$ & $0.0013(8)$ \\
N2 & $0.0270(8)$ & $0.0193(8)$ & $0.0203(8)$ & $0.0014(7)$ & $0.0035(6)$ & $0.0004(7)$ \\
C3 & $0.0337(11)$ & $0.0211(11)$ & $0.0238(9)$ & $0.0053(9)$ & $0.0015(8)$ & $-0.0009(8)$ \\
C4 & $0.0189(9)$ & $0.0188(10)$ & $0.0274(9)$ & $0.0003(8)$ & $-0.0004(7)$ & $-0.0040(8)$ \\
C5 & $0.0181(8)$ & $0.0193(10)$ & $0.0211(9)$ & $-0.0023(8)$ & $0.0016(7)$ & $-0.0021(8)$ \\
O6 & $0.0199(7)$ & $0.0264(8)$ & $0.0243(7)$ & $-0.0044(6)$ & $0.0020(5)$ & $-0.0070(6)$ \\
N7 & $0.0172(8)$ & $0.0195(8)$ & $0.0348(9)$ & $-0.0011(7)$ & $0.0004(6)$ & $-0.0052(8)$
\end{tabular}




$\begin{array}{lllllll}\text { C8 } & 0.0184(9) & 0.0259(10) & 0.0249(9) & 0.0006(8) & 0.0006(7) & -0.0016(9) \\ \text { O9 } & 0.0223(7) & 0.0384(10) & 0.0445(9) & -0.0081(7) & 0.0049(6) & -0.0110(8) \\ \text { C10 } & 0.0229(10) & 0.0357(13) & 0.0474(13) & 0.0049(10) & 0.0051(9) & -0.0145(12) \\ \text { C11 } & 0.0337(11) & 0.0248(11) & 0.0230(9) & -0.0006(9) & 0.0074(8) & -0.0002(9) \\ \text { C12 } & 0.0265(10) & 0.0269(10) & 0.0200(8) & 0.0000(9) & 0.0072(7) & -0.0008(9) \\ \text { C13 } & 0.0404(13) & 0.0400(14) & 0.0325(12) & 0.0081(12) & -0.0079(10) & -0.0066(11) \\ \text { C14 } & 0.0498(15) & 0.0455(17) & 0.0424(14) & 0.0198(13) & -0.0066(12) & -0.0015(13) \\ \text { C15 } & 0.0488(14) & 0.0281(13) & 0.0413(13) & 0.0058(12) & 0.0158(11) & -0.0026(11) \\ \text { C16 } & 0.0353(12) & 0.0386(14) & 0.0466(14) & -0.0049(12) & 0.0061(11) & -0.0150(12) \\ \text { C17 } & 0.0282(10) & 0.0369(13) & 0.0372(12) & 0.0021(11) & -0.0006(9) & -0.0113(11) \\ \text { C18 } & 0.0281(10) & 0.0176(10) & 0.0236(9) & -0.0020(8) & 0.0012(7) & 0.0001(8) \\ \text { O19 } & 0.0322(8) & 0.0176(7) & 0.0284(7) & 0.0028(6) & 0.0031(6) & 0.0046(6) \\ & & & & & \end{array}$

Geometric parameters $\left(\AA,{ }^{\circ}\right)$

\begin{tabular}{|c|c|c|c|}
\hline $\mathrm{C} 1-\mathrm{N} 2$ & $1.489(2)$ & $\mathrm{C} 10-\mathrm{H} 102$ & 0.963 \\
\hline $\mathrm{C} 1-\mathrm{C} 5$ & $1.540(3)$ & $\mathrm{C} 10-\mathrm{H} 103$ & 0.972 \\
\hline $\mathrm{C} 1-\mathrm{C} 18$ & $1.527(3)$ & $\mathrm{C} 11-\mathrm{C} 12$ & $1.510(3)$ \\
\hline $\mathrm{C} 1-\mathrm{H} 11$ & 1.000 & $\mathrm{C} 11-\mathrm{H} 111$ & 0.983 \\
\hline $\mathrm{N} 2-\mathrm{C} 3$ & $1.470(3)$ & $\mathrm{C} 11-\mathrm{H} 112$ & 0.978 \\
\hline $\mathrm{N} 2-\mathrm{C} 11$ & $1.467(3)$ & $\mathrm{C} 12-\mathrm{C} 13$ & $1.379(3)$ \\
\hline $\mathrm{C} 3-\mathrm{C} 4$ & $1.523(3)$ & $\mathrm{C} 12-\mathrm{C} 17$ & $1.391(3)$ \\
\hline $\mathrm{C} 3-\mathrm{H} 31$ & 0.993 & $\mathrm{C} 13-\mathrm{C} 14$ & $1.391(4)$ \\
\hline C3-H32 & 0.970 & C13-H131 & 0.972 \\
\hline $\mathrm{C} 4-\mathrm{C} 5$ & $1.535(3)$ & $\mathrm{C} 14-\mathrm{C} 15$ & $1.378(4)$ \\
\hline $\mathrm{C} 4-\mathrm{N} 7$ & $1.446(3)$ & $\mathrm{C} 14-\mathrm{H} 141$ & 0.993 \\
\hline $\mathrm{C} 4-\mathrm{H} 41$ & 0.976 & $\mathrm{C} 15-\mathrm{C} 16$ & $1.382(4)$ \\
\hline $\mathrm{C} 5-\mathrm{O} 6$ & $1.427(2)$ & $\mathrm{C} 15-\mathrm{H} 151$ & 0.979 \\
\hline $\mathrm{C} 5-\mathrm{H} 51$ & 0.989 & $\mathrm{C} 16-\mathrm{C} 17$ & $1.384(4)$ \\
\hline $\mathrm{O} 6-\mathrm{H} 17$ & 0.803 & $\mathrm{C} 16-\mathrm{H} 161$ & 0.978 \\
\hline $\mathrm{N} 7-\mathrm{C} 8$ & $1.337(2)$ & $\mathrm{C} 17-\mathrm{H} 171$ & 0.981 \\
\hline $\mathrm{N} 7-\mathrm{H} 8$ & 0.838 & $\mathrm{C} 18-\mathrm{O} 19$ & $1.418(2)$ \\
\hline $\mathrm{C} 8-\mathrm{O} 9$ & $1.235(3)$ & $\mathrm{C} 18-\mathrm{H} 181$ & 0.995 \\
\hline $\mathrm{C} 8-\mathrm{C} 10$ & $1.500(3)$ & $\mathrm{C} 18-\mathrm{H} 182$ & 0.979 \\
\hline $\mathrm{C} 10-\mathrm{H} 101$ & 0.965 & $\mathrm{O} 19-\mathrm{H} 15$ & 0.925 \\
\hline $\mathrm{N} 2-\mathrm{C} 1-\mathrm{C} 5$ & $105.3(2)$ & $\mathrm{H} 101-\mathrm{C} 10-\mathrm{H} 102$ & 106.6 \\
\hline $\mathrm{N} 2-\mathrm{C} 1-\mathrm{C} 18$ & $108.5(2)$ & $\mathrm{C} 8-\mathrm{C} 10-\mathrm{H} 103$ & 113.3 \\
\hline $\mathrm{C} 5-\mathrm{C} 1-\mathrm{C} 18$ & $111.6(2)$ & $\mathrm{H} 101-\mathrm{C} 10-\mathrm{H} 103$ & 109.0 \\
\hline $\mathrm{N} 2-\mathrm{C} 1-\mathrm{H} 11$ & 111.50 & $\mathrm{H} 102-\mathrm{C} 10-\mathrm{H} 103$ & 107.3 \\
\hline $\mathrm{C} 5-\mathrm{C} 1-\mathrm{H} 11$ & 109.3 & $\mathrm{~N} 2-\mathrm{C} 11-\mathrm{C} 12$ & $112.1(2)$ \\
\hline $\mathrm{C} 18-\mathrm{C} 1-\mathrm{H} 11$ & 110.6 & $\mathrm{~N} 2-\mathrm{C} 11-\mathrm{H} 111$ & 109.9 \\
\hline $\mathrm{C} 1-\mathrm{N} 2-\mathrm{C} 3$ & $108.5(2)$ & $\mathrm{C} 12-\mathrm{C} 11-\mathrm{H} 111$ & 108.6 \\
\hline $\mathrm{C} 1-\mathrm{N} 2-\mathrm{C} 11$ & $114.6(2)$ & $\mathrm{N} 2-\mathrm{C} 11-\mathrm{H} 112$ & 108.4 \\
\hline $\mathrm{C} 3-\mathrm{N} 2-\mathrm{C} 11$ & $112.9(2)$ & $\mathrm{C} 12-\mathrm{C} 11-\mathrm{H} 112$ & 109.4 \\
\hline $\mathrm{N} 2-\mathrm{C} 3-\mathrm{C} 4$ & $101.2(2)$ & H111-C11-H112 & 108.4 \\
\hline $\mathrm{N} 2-\mathrm{C} 3-\mathrm{H} 31$ & 112.5 & $\mathrm{C} 11-\mathrm{C} 12-\mathrm{C} 13$ & $120.9(2)$ \\
\hline $\mathrm{C} 4-\mathrm{C} 3-\mathrm{H} 31$ & 110.9 & $\mathrm{C} 11-\mathrm{C} 12-\mathrm{C} 17$ & $120.7(2)$ \\
\hline
\end{tabular}




\begin{tabular}{|c|c|c|c|}
\hline $\mathrm{N} 2-\mathrm{C} 3-\mathrm{H} 32$ & 110.5 & $\mathrm{C} 13-\mathrm{C} 12-\mathrm{C} 17$ & $118.4(2)$ \\
\hline $\mathrm{C} 4-\mathrm{C} 3-\mathrm{H} 32$ & 112.6 & $\mathrm{C} 12-\mathrm{C} 13-\mathrm{C} 14$ & $120.9(2)$ \\
\hline $\mathrm{H} 31-\mathrm{C} 3-\mathrm{H} 32$ & 109.0 & $\mathrm{C} 12-\mathrm{C} 13-\mathrm{H} 131$ & 119.3 \\
\hline $\mathrm{C} 3-\mathrm{C} 4-\mathrm{C} 5$ & $101.7(2)$ & $\mathrm{C} 14-\mathrm{C} 13-\mathrm{H} 131$ & 119.8 \\
\hline $\mathrm{C} 3-\mathrm{C} 4-\mathrm{N} 7$ & $114.8(2)$ & $\mathrm{C} 13-\mathrm{C} 14-\mathrm{C} 15$ & $120.3(2)$ \\
\hline $\mathrm{C} 5-\mathrm{C} 4-\mathrm{N} 7$ & $114.0(2)$ & $\mathrm{C} 13-\mathrm{C} 14-\mathrm{H} 141$ & 119.7 \\
\hline $\mathrm{C} 3-\mathrm{C} 4-\mathrm{H} 41$ & 109.3 & $\mathrm{C} 15-\mathrm{C} 14-\mathrm{H} 141$ & 119.9 \\
\hline $\mathrm{C} 5-\mathrm{C} 4-\mathrm{H} 41$ & 109.0 & $\mathrm{C} 14-\mathrm{C} 15-\mathrm{C} 16$ & $119.1(2)$ \\
\hline $\mathrm{N} 7-\mathrm{C} 4-\mathrm{H} 41$ & 107.8 & $\mathrm{C} 14-\mathrm{C} 15-\mathrm{H} 151$ & 120.5 \\
\hline $\mathrm{C} 1-\mathrm{C} 5-\mathrm{C} 4$ & $102.1(2)$ & $\mathrm{C} 16-\mathrm{C} 15-\mathrm{H} 151$ & 120.4 \\
\hline $\mathrm{C} 1-\mathrm{C} 5-\mathrm{O} 6$ & $114.0(2)$ & $\mathrm{C} 15-\mathrm{C} 16-\mathrm{C} 17$ & $120.5(2)$ \\
\hline $\mathrm{C} 4-\mathrm{C} 5-\mathrm{O} 6$ & $110.2(2)$ & $\mathrm{C} 15-\mathrm{C} 16-\mathrm{H} 161$ & 119.9 \\
\hline $\mathrm{C} 1-\mathrm{C} 5-\mathrm{H} 51$ & 111.2 & $\mathrm{C} 17-\mathrm{C} 16-\mathrm{H} 161$ & 119.6 \\
\hline $\mathrm{C} 4-\mathrm{C} 5-\mathrm{H} 51$ & 111.1 & $\mathrm{C} 12-\mathrm{C} 17-\mathrm{C} 16$ & $120.7(2)$ \\
\hline $\mathrm{O} 6-\mathrm{C} 5-\mathrm{H} 51$ & 108.2 & $\mathrm{C} 12-\mathrm{C} 17-\mathrm{H} 171$ & 118.6 \\
\hline $\mathrm{C} 5-\mathrm{O} 6-\mathrm{H} 17$ & 113.1 & $\mathrm{C} 16-\mathrm{C} 17-\mathrm{H} 171$ & 120.7 \\
\hline $\mathrm{C} 4-\mathrm{N} 7-\mathrm{C} 8$ & $122.4(2)$ & $\mathrm{C} 1-\mathrm{C} 18-\mathrm{O} 19$ & $113.7(2)$ \\
\hline $\mathrm{C} 4-\mathrm{N} 7-\mathrm{H} 8$ & 117.0 & $\mathrm{C} 1-\mathrm{C} 18-\mathrm{H} 181$ & 107.7 \\
\hline $\mathrm{C} 8-\mathrm{N} 7-\mathrm{H} 8$ & 120.4 & $\mathrm{O} 19-\mathrm{C} 18-\mathrm{H} 181$ & 108.5 \\
\hline $\mathrm{N} 7-\mathrm{C} 8-\mathrm{O} 9$ & $121.9(2)$ & $\mathrm{C} 1-\mathrm{C} 18-\mathrm{H} 182$ & 109.2 \\
\hline $\mathrm{N} 7-\mathrm{C} 8-\mathrm{C} 10$ & $116.6(2)$ & $\mathrm{O} 19-\mathrm{C} 18-\mathrm{H} 182$ & 108.7 \\
\hline $\mathrm{O} 9-\mathrm{C} 8-\mathrm{C} 10$ & $121.5(2)$ & $\mathrm{H} 181-\mathrm{C} 18-\mathrm{H} 182$ & 108.9 \\
\hline $\mathrm{C} 8-\mathrm{C} 10-\mathrm{H} 101$ & 111.5 & $\mathrm{C} 18-\mathrm{O} 19-\mathrm{H} 15$ & 111.2 \\
\hline $\mathrm{C} 8-\mathrm{C} 10-\mathrm{H} 102$ & 108.9 & & \\
\hline
\end{tabular}

Hydrogen-bond geometry $\left(A,{ }^{\circ}\right)$

\begin{tabular}{lllll}
\hline$D-\mathrm{H}^{\prime} \cdots A$ & $D-\mathrm{H}$ & $\mathrm{H} \cdots A$ & $D \cdots A$ & $D-\mathrm{H} \cdots A$ \\
\hline $\mathrm{N} 7-\mathrm{H} 8 \cdots \mathrm{O} 19^{\mathrm{i}}$ & 0.84 & 2.14 & $2.958(2)$ & 167 \\
$\mathrm{O} 19-\mathrm{H} 15 \cdots 6^{\mathrm{ii}}$ & 0.93 & 1.85 & $2.708(2)$ & 153 \\
$\mathrm{O} 6-\mathrm{H} 17 \cdots \mathrm{O}^{\mathrm{iii}}$ & 0.80 & 1.89 & $2.685(2)$ & 168 \\
\hline
\end{tabular}

Symmetry codes: (i) $x, y-1, z$; (ii) $-x+1, y+1 / 2,-z+1$; (iii) $x-1, y, z$. 